\title{
Three stages Interactive System for Solving Children Communication Disorder
}

\author{
W.M. Shalash \\ Information Technology \\ department, King Abdul Aziz \\ University \\ P.O Box 42808, Jeddah \\ 21551, Saudi Arabia
}

\author{
Noura Sait \\ Information Technology \\ department, King Abdul Aziz \\ University \\ P.O Box 42808, Jeddah \\ 21551, Saudi Arabia
}

\author{
Duaa Hashem \\ Information Technology \\ department, King Abdul Aziz \\ University \\ P.O Box 42808, Jeddah \\ 21551, Saudi Arabia
}

\begin{abstract}
The great advance in information technology during the last two decades contributes significantly in solving special needed people problems. This paper describes the second phase of an ongoing project to help those children suffering from speech disorder problems. The current application is a multi-levels interactive game using speech recognition technology; kid can interact with an interactive animated picture using his/her voice. There are three levels for improving the speaking skills of children at different stages. Firstly, by encouraging child just to produce sounds then by improving his/her pronunciation skills by pronouncing short vocal (one syllable words)Arabic words and finally improving his/her skill by pronouncing long vocal (for example two syllable words). This application solves speech disorder problems such as difficulty producing speech, dysfluency, shuttering and voice disorders. The current work is pioneer in developing an Arabic language application and targeted to children aged from two years to seven years.
\end{abstract}

\section{General Terms}

Human-computer Interaction, Speech disorder therapy.

\section{Keywords}

Children speech disorder, speech recognition, interactive applications.

\section{INTRODUCTION}

Children speech or language disorder can be considered as a critical problem not only affecting the child and his own family but also the whole society. If the child doesn't receive the proper therapy in the proper time he/she may suffering from that problem the rest of his/her life not only on educational performance but on his/her opportunity to get good jobs and to communicate with the whole society[1].

The concept of communication disorders comprises a wide variety of problems in language, speech, and hearing. Speech and language impairments include articulation problems, voice disorders, fluency problems (such as stuttering), aphasia (difficulty in using words, sometimes as a result of a brain injury, viral infections, cardio-vascular accident, mental retardation), and delays in speech and/or language. Hearing impairments include partial hearing and deafness. Deafness refers to a kind of loss sufficient to make auditory communication difficult or impossible without amplification. A good number of communication disorders can equally result from other pathological conditions such as learning disabilities, cerebral palsy, mental retardation, or cleft lip or cleft palate [2].
Different researchers have studied speech and language dysfunctions depending on their disciplines and orientations. Not all speech variations are clinical problems. A speech difference is a problem if it is interferes with communicabilities attracts undue or negative, attention or causes the speaker to be emotionally disturbed or socially maladjusted [3].

Freeman \& Silver [4] had noted that distinguished speech disorders are typically more difficult in children than adults, and most difficult in cerebral palsied subjects. However, these children are not placed in treatment for speech disorder until it was clear that they were not going to outgrow the problem in addition to limited professional and financial resources. As consequence, most subjects did not receive professional assistance until they were over 7 years old. In these older subjects, deviant speech patterns were firmly established [5].

Children suffering from communication disorders tend to manifest a number of peculiar cognito-behavioural features. For instance, a child with speech or language delays may present a variety of characteristics including the inability to follow directions, slow and incomprehensible speech, and pronounced difficulties in syntax and articulation. Articulation disorders are characterized by the substitution of one sound for another or the omission or distortion of certain sounds. Stuttering or dysfluency is a disorder of speech flow that most often appears between the ages of 3 and 4 years and may progress from a sporadic to a chronic problem. Stuttering may spontaneously disappear by early adolescence, but speech and language therapy should be considered. Typical voice disorders include hoarseness, breathiness, or sudden breaks in loudness or pitch. Voice disorders are frequently combined with other speech problems to form a complex communication disorder [2].

"Speech or Language Impairment" means a communication disorder, such as stuttering, impaired articulation, language impairment, or a voice impairment, that adversely affects a child's educational performance [5].

\subsection{Types of Speech Disorder}

Speech disorder has many types including:

- Dysfluency: an interruption in the flow of speech, such as stuttering e.g. some person who stutters may repeat the first part of a word (as in wa-wa-wa-water) or hold a single sound for a long time (as in caaaaaaake).

- Articulation or phonological disorders: difficulties with the pitch, volume or quality of the voice while the sound is formed e.g. Substituting a "w" for an "r" ("wabbit" for "rabbit"), omitting sounds ("cool" for "school"), or 
adding sounds to words ("pinanio" for "piano") are examples of articulation errors.

- Aphasia: is the loss of the ability to understand language, whether spoken or written, and occurs due to disturbances in the areas of the brain that are used in language processing.

- Dysarthria: Dysarthria refers to a difficulty in pronouncing certain sounds or words that is usually due to a problem with muscle control.

- Dyspraxia: is a motor learning difficulty that can affect planning of movements and co-ordination as a result of brain messages not being accurately transmitted to the body.

- Slurred Speech :speech in which words are not enunciated clearly or completely but are run together or partially eliminated

- Speech Disturbance: Difficulty with speech can be the result of problems with the brain or nerves that control the facial muscles, larynx, and vocal cords necessary for speech.

- $\quad$ Speech Impediment.

- The Language Disorders is the situation that the child has a large amount of vocabulary but he/she can't use them at sentences and doesn't use the grammatical patterns for making sentences.

While Types of Language Disorders includes:

- $\quad$ First type: improper use of words and their meanings.

- Second type: inability to express ideas, inappropriate grammatical patterns.

Third type: reduced vocabulary and inability to follow directions.

As mentioned in the above sections speech disorder is a critical and important problem and it is better to find solutions for it in early stages to overcome its drawbacks. Therefore, in the current work we tried to introduce an easy, cheap, and interactive solution for the families have child suffering from this problem and also for therapists.

The current work is an Arabic language application based on the idea that, a particular beneficial feature of humancomputer interaction (HCI) is that children find them a "safe" and enjoyable experience. This can be explained by the fact that interaction with computers does not pose the expectations and judgment issues that are associated with social interaction. Computer systems tend to function in a controlled environment with minimal distractions and this makes them an attractive option for the education of autistic children [6]. Consequently, this type of interaction elicits positive feelings, whereas communication with humans is frequently fraught with problems [7]. The positive feelings appear to be generic and uncorrelated with the type of software interface [8].

The current work training strategy followed the suggested strategy illustrated on [5] to start by training speech muscles: by slow abdominal respiration to increase expiration and use air amount for speaking; first for voices then letters then words then sentence [5].

The current has been started in [1] with the first two phases and currently by adding the third stage to complete it. The current work is targeted to children ages from 2 years to 7 years.

\section{RELATED WORK}

Many commercial application deal with speech disorder problems such as Speech-Language Pathologist Package[9], Bungalow Software [10], Dr. Speech [11] and Say It Best speech Articulation[12]

Regarding to Arabic reading applications for communication disorder therapy, there are relatively few software applications that are targeting speech disorder problems.

\section{METHODOLOGY}

The proposed application consists of three levels in order to help in children speech disorder therapy. This application aims to help kids to learn and improve their communication skills in an easy, interactive manner, like playing a game. The current application main advantage is that it is in Arabic where most of the available commercial applications are in English. This application can be used easily at home, institution or any place. It also helps adults (therapists or parents) to keep tracking of the child progress by recording his/her score each level.

The animated picture and speech recognition technologies have been combine in order to develop the current system. Children usually enjoy therapy with colorful, interactive, game-like software because they receive immediate animated feedback on their performance. The current system consists of three levels. These three levels will be illustrated in the following sub-sections.

\subsection{Level One}

The first level is very basic to help child to improve his/her speech skills and making sure that the child does not have any health problem preventing him from specking. This level aims to encourage the child to produce sounds. It provides an interesting interactive interface showing friendly simple objects. These objects start to animate as the child produces sounds and this behavior changes when sound threshold level increased in an interesting manner.

As an example, one interface story contains a car starts to move as a child starts to produce sounds and it will stop if he/she stops producing sound. Then, it changes to become a 2 racing cars as the child increases his/her voice (as shown if figure 1), if the child decrease his/her sound level the cars speed will reduced and if he stop the cars will stop moving. Figure 2 and 3 show another two stories just like the first figure but with different animated object popular to children.

\subsection{Level Two}

The second level is devoted to those children who got the ability to pronounce letters but in a wrong way. This level aims to correct their language disorder problems by providing words with related pictures and encourage them to pronounce words correctly by producing clapping sounds, saying words in Arabic language such as "Good job" and rewarding children by giving them marks if they saying word correctly. This level contains two stages one of short vocal (one syllable words) words such as cat or beer in Arabic (دب - قطه) while, the other stage contains long vocal (two syllable words) such as lemon or apple in Arabic(ليمون - تفاح). This level based on a simple speech recognition system consists of pre-processing stage (Butter worth band-pass filter) for noise removal followed by Dynamic time warpping and feature extraction stage using Mel frequency Cepstral coefficient, finally 
performing classification stage by comparing the distance between input features with the saved corresponding one from system database as shown in figure 4 [13 - 15]. Figure 5 and 6 show a sample screenshot from level 2 interface.

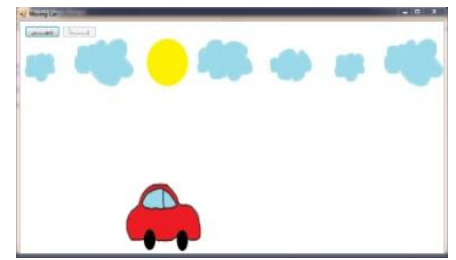

(a)

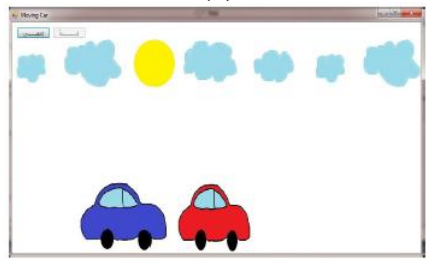

(b)

Fig 1: Sample screenshots of the first level interactive interfaces (car story), a)when child start producing sound a racing car appears and $b$ ) when sound level increased another car start racing also

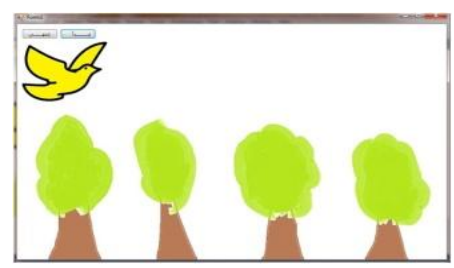

(a)

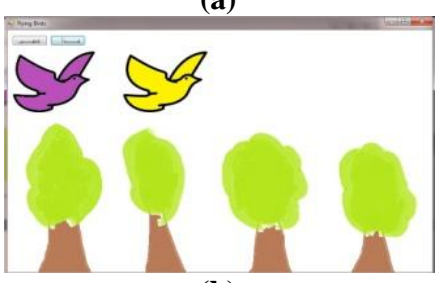

(b)

Fig 2: Sample screenshots of the first level interactive interfaces (bird story), a)when child start producing sound a flying bird appears and b) when sound level increased another flying bird comes

\subsection{Level Three}

The third level designed for improving the communication skills by involving the kids into a conversation and asking them to complete a task in a right way. For example, one of the third level stories is that the mother enters the child messy room and point to the unordered toys and if he/she pronounce it correctly, the toy will be placed on its correct place as shown in figure 7.

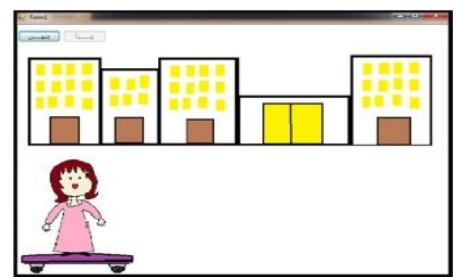

(a)

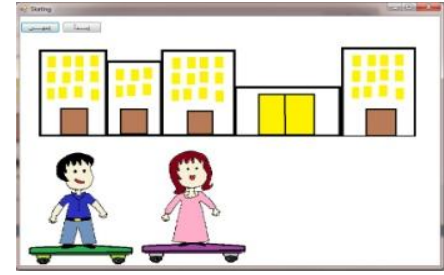

(b)

Fig 3: Sample screenshots of the first level interactive interfaces (girl and boy story), a) when child start producing sound a girl appears ridding scoter and b) when sound level increased a boy appears and racing the girl

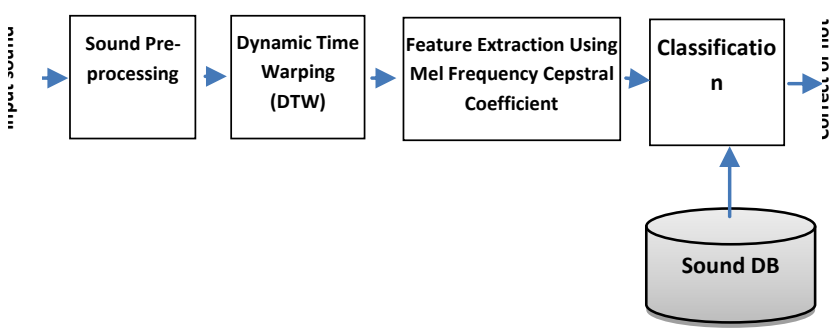

Fig 4: Level 2 simple speech recognition system block diagram

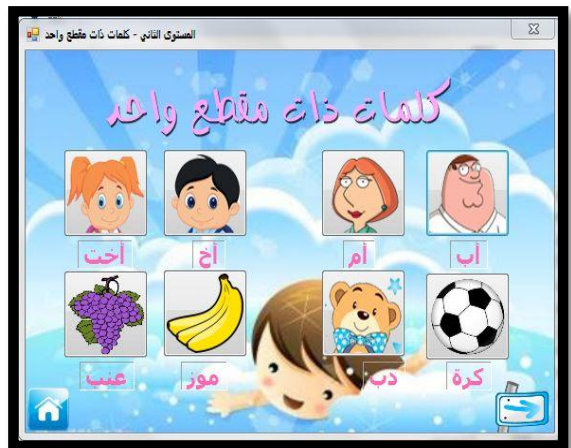

Fig 5: Sample screenshots of level two interactive interfaces (one-syllable words) main interface (in Arabic)

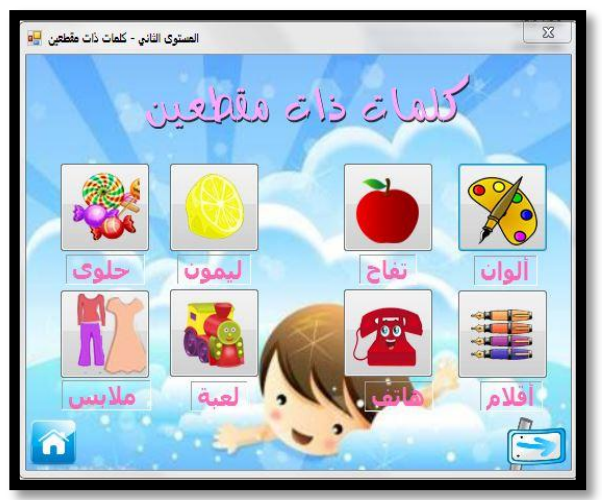

Fig 6: A sample screenshot for the second level, stage two (two syllable words) main interface (in Arabic). 


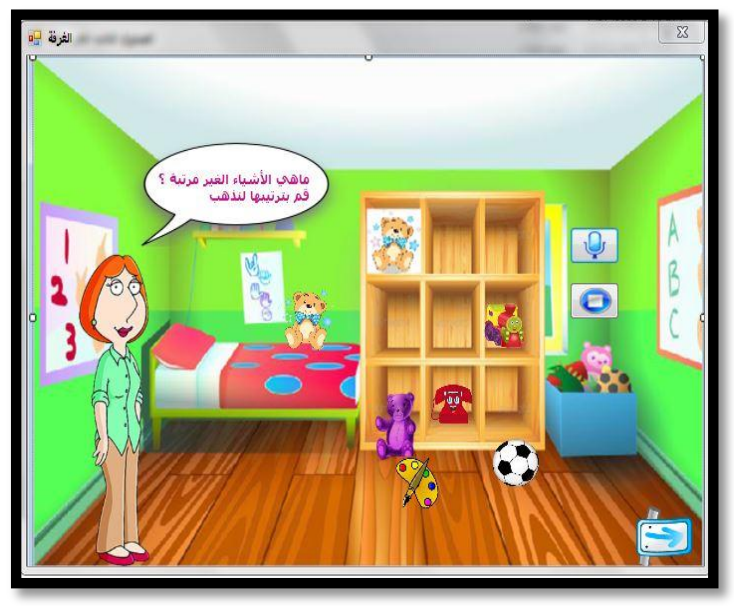

Fig 7: Level 3, Messy room story.

\section{RESULTS AND DISCUSSION}

The implementation and test of the current work has been performed into two phases on the first phase we just implement and test the first level and one part of the second one (one syllable words) as reported on [1]. While in the second phase, we implement and test the complete three stages and improve the animated graphics by using Adobe Flash with action script 3 . Tracking records for the child have been added. These records enable the therapist and child parents to follow the child progress.

The usability test was conducted on two groups of users 5 therapists and 15 parents as one group and 15 children their ages ranges from two and half years to seven years as second group. The tests started by giving the each group (therapists, parents and children) an overview about our application, and then giving them tasks to perform. While the most three important parts of the system are Registration (for therapists and parents), playing level one, two and three (for children), the testing process will focus on those three parts. Testers observed tests and recorded information about time needed to perform each task and the number of correct clicks and wrong clicks. An interview has been conducted with therapists, parents and children about the software application and the problems they faced after using it.

\subsubsection{The following are the tasks given to users}

Giving a certain users 6 tasks for testing our application that include:

1. Create an account that contains your information as supervisor's account.

2. Log in to your account that you had create it in pervious task

3. Add a child with this information: ( name : Mariam Amrou Sif ,gender : girl, birthday 11/8/2005, city : Jeddah, address : Al-Rawdah neighborhood, Phone No. : 02-6666776 ).

4. Make the child that you registered before start playing with the level that measure the child's ability to issue votes (Level one).

5. Make the child that you registered before start playing with the level that tests the child pronunciation (Level two)
6. Make the child that you registered before start playing with the level that tests the child pronunciation (Level three).

7. View the child statues from the children.

After observation of the testing process, the results of the usability test shows that all of 5 therapists and 13 of 15 of the parents did all the tasks directly and navigate through the application windows easily without mistakes. The children test results shows that for the first level 14 of 15 children play it in a short time, while in the second level test 12 of 15 play it in a short time. The result of level three testing also shows that 11 of 15 play it in a correct way. Figure 8 and 9 show group 1 and 2 usability test results. Generally, the children impression was good and they like the animated pictures response a lot.

The results of the usability test show promising results for both speech therapist and kids. Most of the users performed the required task in a reasonable time and number of clicking.

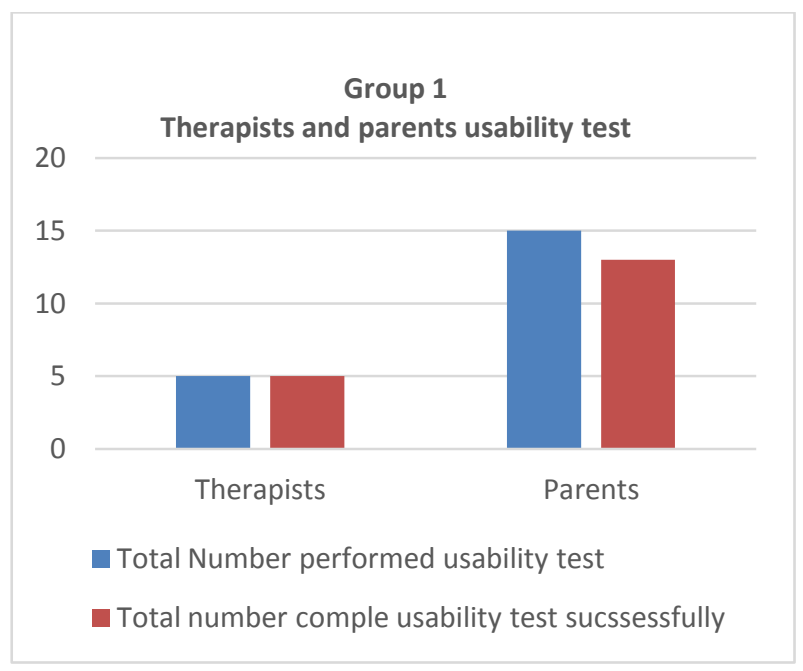

Fig 8: Group 1 usability test results.

After task session completion, participants of first group (therapists and parents) rated the System by six overall measures, these measures include:

1. Ease of use

2. Clarity of the buttons meaning.

3. Clarity of menus and its contents.

4. Clarify of help windows

5. The quality and consistency of colors and fonts.

Table-1 shows analysis of the results from the post-test questionnaire.

Table 1. Analysis of post-test questionnaire

\begin{tabular}{|c|l|c|}
\hline Question & Answer & Average \\
\hline $\begin{array}{c}\text { Using the } \\
\text { software was }\end{array}$ & Very easy & $80 \%$ \\
\cline { 2 - 3 } & Easy & $14 \%$ \\
\cline { 2 - 3 } & Neither easy nor difficult & $0 \%$ \\
\cline { 2 - 3 } & Difficult & $7 \%$ \\
\hline
\end{tabular}




\begin{tabular}{|c|c|c|}
\hline & Very difficult & $0 \%$ \\
\hline \multirow{3}{*}{$\begin{array}{l}\text { Clarity of } \\
\text { button } \\
\text { meanings }\end{array}$} & Very clear and understandable & $73 \%$ \\
\hline & Clear and somewhat & $14 \%$ \\
\hline & Not clear & $13 \%$ \\
\hline \multirow{3}{*}{$\begin{array}{c}\text { Clarity } \\
\text { menus and its } \\
\text { contents }\end{array}$} & Very clear and understandable & $93 \%$ \\
\hline & Somewhat clear & $7 \%$ \\
\hline & Not clear & $0 \%$ \\
\hline \multirow{3}{*}{$\begin{array}{c}\text { Clarity of } \\
\text { help windows }\end{array}$} & Very clear and understandable & $100 \%$ \\
\hline & Somewhat clear & $0 \%$ \\
\hline & Not clear & $0 \%$ \\
\hline \multirow{3}{*}{$\begin{array}{l}\text { The quality } \\
\text { and } \\
\text { consistency } \\
\text { of fonts and } \\
\text { colors }\end{array}$} & Comfortable and consistent & $80 \%$ \\
\hline & $\begin{array}{l}\text { Somewhat Comfortable and } \\
\text { consistent }\end{array}$ & $20 \%$ \\
\hline & $\begin{array}{l}\text { Inconsistent and } \\
\text { Uncomfortable }\end{array}$ & $0 \%$ \\
\hline
\end{tabular}

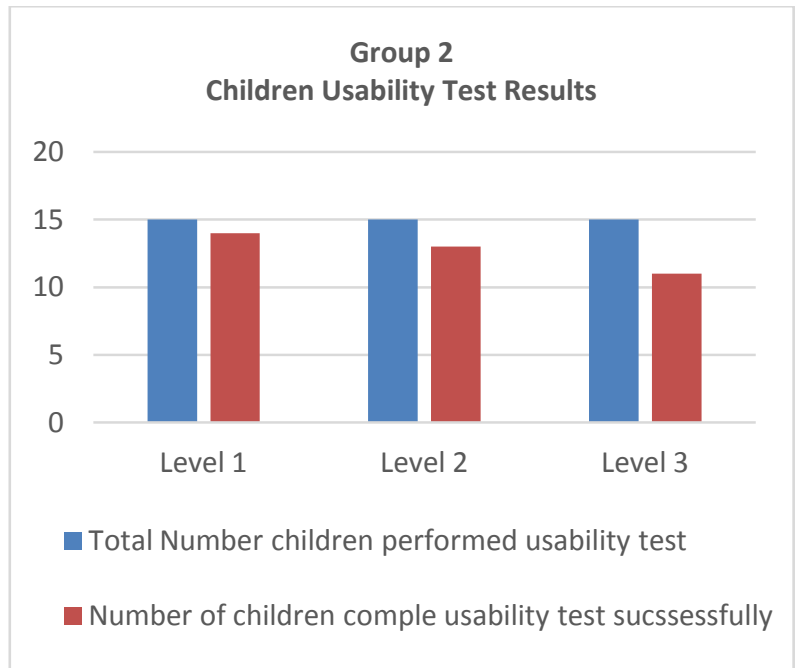

Fig 9: Group 2 (Children) usability test results

\section{CONCLUSION}

This paper presents an ongoing project to produce a software package to be used in the thereby of those children suffering from speech disorder in an interactive game manner. The current work covers three stages in our way to improve children communication skills. The first stage was concerning of encourage children to produce sounds, while the second stage improving the pronunciation of letters through one syllable words followed by two syllable words in an interactive manner. Finally, the third stage tried to improve child communication skill through long interactive stories combining animated cartoon characters with sound recognition to produce dialog system. A record for tracing each child score through levels has been provided in order to help therapists and parents watching the child progress.

The main advantage of this application is that it is in Arabic where most of the applications available in market are in English. This application can be used easily at home, institution or any place so, it is an inexpensive way to overcome speech disorder problem.
We achieved a great progress in completing our work that has been started in [1]. In near future we aim to complete to produce dialog system and to increase number of words in each level combining animation with virtual reality application. We also aim to test the system with much more number of children, applying more analysis with respect to age and gender.

\section{ACKNOWLEDGMENTS}

Our thanks to Malak Bas-sam and Ghada Shawly for their contribution in the development of the project first phase. Special grateful thanks for Hadel Beama who implement all the animated graphs of the second phase.

\section{REFERENCES}

[1] Wafaa M. Shalash, Malak Bas-sam, Ghada Shawly, Interactive System for Solving Children Communication Disorder, pp 462-469,Second International Conference, DUXU 2013, Held as Part of HCI International 2013, Las Vegas, NV, USA, July 21-26, 2013 .DOI: 10.1007/978-3-642-39241-2_51

[2] C. U. Agbedo. 2008 Communication Disorders in Children: A Case Study of Mimo Usama and Sele Yengi, International Journal of Communication: An Interdisciplinary Journal of Communication Studies, Vol. 8, No. 1 (2008).

[3] Daniloff, R. (Ed.). 1984 Articulation assessment and treatment issues, College Hill press, sanDiego, CA, 247(1984).

[4] Freeman, F. and L. Silver.1989 Developmental Articulation Disorders compr. Textb of psychiat II, chap. 36. 3a, pp. 1804 -9 H. Kaplan \& B. Sadock (Eds.).

[5] Nawal M. Khalifa, Elfiky, M.1 2 R. and 3Basiony, S. 2008 Research Journal of Medicine and Medical Sciences, 4(1): 7-13( 2008).

[6] Green SJ. 1993 Computer-based simulations in the education and assessment of autistic children. In: Rethinking the Roles of Technology in Education, Tenth International Conference on Technology and Education, Cambridge, MA, Massachusetts Institute of Technology, pp. 334-36, Volume 1(1993).

[7] Hutinger P, Rippey R. : How five preschool children with autism responded to computers, http://scott.mprojects.wiu.edu/ eccts/articles/autism1.ht $\mathrm{ml}$.

[8] Evdokimos Konstantinidis, Andrej Luneski, Christos Frantzidis, Maria Nikolaidou, Magda HitoglouAntoniadou†, Panagiotis Bamidis, 2009. Information and Communication Technologies (ICT) for Enhanced Education of Children with Autism Spectrum Disorders, The Journal on Information Technology in Healthcare; 7(5), pp 284-292(2009).

[9] Speical needs software, 2011.Speech-Language Pathologist Package, http://www.laureatelearning.com/specials/slppack.html.

[10] [Bungalow Software http://www.bungalowsoftware.com.

2010.

[11] Dr.Speech Software(1990), http://www.drspeech.com.

[12] Say It Best Speech Articulation Software, 2012 http://www.sayitbest.info/speech-therapy-games-forkids/Articulation-Software-Game-Research.html 
[13] Juang, Biing-hwang Hwang and Wu hou;Lee, 1997. Minimum classification error rate methods for speech recognition, IEEE Transactions on Speech and Audio Processing, Page(s) 257 - 265, Issue 3 Volume 5(1997).

[14] Molau, Sirko Lehrstuhl fur Inf, Pitz, Michael; Schluter, R.; Ney, Herrmann J.: Computing Mel-frequency cepstral coefficients on the power spectrum, Page(s) 73 -
76 vol.1, IEEE International Conference on Acoustics, Speech, and Signal Processing, (ICASSP '01) ( 2001) .

[15] Stan Salvador, Chan, Fast DTW, 2007 Toward Accurate Dynamic Time Warping in Linear Time and Space, IEEE Transactions on Biomedical. Engineering, vol. 43, no. 4(2007). 